\title{
Compiling REAPER (Robust Epoch And Pitch EstimatoR)
}

Cong Zhang, 28/5/2021

This is a brief tutorial for compiling and installing REAPER (David Talkin, https://github.com/goog! e/REAPER).

If you have never used $\mathrm{C} / \mathrm{C}++$, it takes some time downloading and configuring the correct things. I hope these procedures are helpful. I would also like to thank all the authors of the tutorials I cited in this tutorial. Without those tutorials, I would never be able to install REAPER. I hope this tutorial benefit others, like those tutorials benefited me.

These procedures are only tested on Windows 10.

\section{Choose a folder for REAPER}

e.g. I chose the Documents folder: C: \Users $\backslash$ congzhang $\backslash$ Documents

2. Open Command Prompt on your computer. You can find it by simply typing $\mathrm{cmd}$ in your start menu. If you don't know how to find it, google "find cmd windows 10".

3. Change the directory to where you want to keep the REAPER folder. In my case, it is cd Documents

4. Follow the instructions on REAPER's github page and do:

git clone https://github.com/google/REAPER.git \# downloading the codes Cd REAPER \# change directory to the REAPER folder mkdir build cd build \# creating a new folder 'build' \# change directory to 'build'

5. Add this path to the environment variable.

C: \Users \sprin\Documents \REAPER \bui $7 d$

If you do not already know how to add paths to the environment variable, here is a useful tutorial.

6. Download cmake here. I downloaded this version: cmake-3.20.3-windows-x86_64.msi .

7. Now you should have a new directory full of .exe files here:

C: \Program Files \CMake \bin

8. Add the above path ( $\mathrm{C}: \backslash$ Program Files $\backslash$ CMake $\backslash$ bin ) to the environment variable. 
9. Download Microsoft Visual Studio (2019)

Follow this tutorial to download and install Microsoft Visual Studio and the relevant tools. The video is for downloading the 2017 version, but it's basically the same for 2019. Install the Desktop development with $\mathrm{C}++$ component. This is quite large. You need at least $7 \mathrm{~GB}$ free space on your computer.

\section{Desktop \& Mobile (4)}

${ }_{+}^{+} \square$ Desktop development with $\mathrm{C}++$

Build modern $\mathrm{C}++$ apps for Windows using tools of your choice, including MSVC, Clang, CMake, or MSBuild.

10. Add these paths to the environment variable.

C: \Program Files (x86) \Microsoft Visual

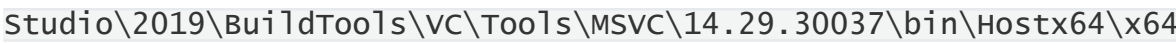

C: $\backslash$ Program Files (x86) \Microsoft Visual

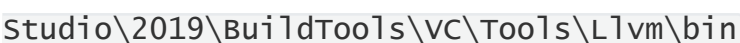

11. Follow this tutorial to download and install MinGW.

The key is to download both mingw32-base and mingw32-gcc-g++ .

Add this path to the path variable:

C: \MinGW \bin

12. Continue with following the instructions on the REAPER github page:

cmake ..

13. If the error message reports some skippings because it cannot find $\mathrm{C}++11$.

Use cmake -G "CodeLite - MinGW Makefiles" .. instead of cmake ..

14. So now you should have these new paths in your environment variable:

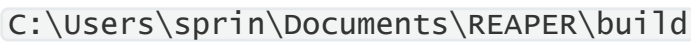

C: \Program Files (x86) \Microsoft Visual

Studio $\backslash 2019 \backslash$ Bui $1 d T$ Too $\backslash s \backslash V C \backslash T o 01 s \backslash M S V C \backslash 14.29 .30037 \backslash$ bin $\backslash$ Hostx64 $\backslash x 64$

C: \Program Files (x86)\Microsoft Visual

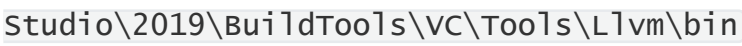

C: \MinGW \bin

C: \Program Files \cMake \bin 
15. Continue with following the instructions on the REAPER github page:

make

16. If you come across an error message about not being able to find 'make.exe'. Follow this tutorial:

- As explained here, create a new file in any of your PATH folders. For example create mingwstartup. bat in the MinGW bin folder. write the line doskey make=mingw32-make. exe inside, save and close it.

- open Registry Editor by running regedit. As explained here in HKEY_LOCAL_MACHINE Or HKEY_CURRENT_USER go to \Software \Microsoft\Command Processor right click on the right panel New --> Expandable String value and name it AutoRun. double click and enter the path

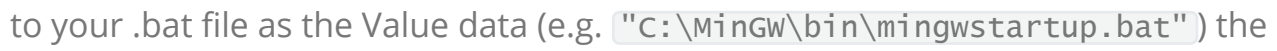
result should look like this:

ab AutoRun "C: REG_EXPAND_SZ MinGM bin \mingwstartup.bat" now every time you open a new terminal make command will run the mingw32make.exe.

17. Always remember to delete this file when you come across errors and have to run the codes

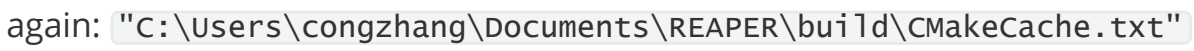

18. The compiling is successful when you have a reaper. exe file in the "C: \Users \sprin\Documents $\backslash$ REAPER $\backslash$ bui $1 d "$ folder.

19. To use the program, you can run something like the following line in the command prompt:

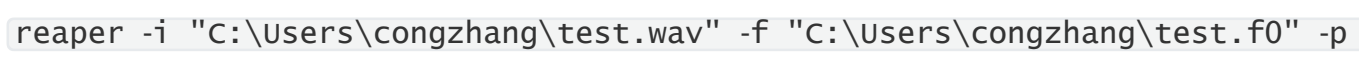
"C:\Users\congzhang\test. pm" -a

The .wav file is the file you want to analyse; the fo file gives you the values of fo;

20. You can use reaper -help to check what other things you can do and what the output files are.

This is where I've got so far. I will update this file when I have more information that I think are helpful.

Dr Cong Zhang cong.zhang@ru.nl

Twitter: @congprosody. 